\title{
Equilibrating C-S Bond Formation by C-H and S-S Bond Metathesis. Rhodium-Catalyzed Alkylthiolation Reaction of 1-Alkynes with Disulfides
}

\author{
Mieko Arisawa, Kenji Fujimoto, Satoshi Morinaka, and Masahiko Yamaguchi*
}

\begin{abstract}
Department of Organic Chemistry, Graduate School of Pharmaceutical Sciences,
\end{abstract}
Tohoku University, Aoba, Sendai, 980-8578, Japan

\begin{abstract}
${ }^{1} \mathrm{H}-\mathrm{NMR}$ and ${ }^{13} \mathrm{C}-\mathrm{NMR}$ spectra were obtained on a Varian Mercury (400 MHz). Chemical shift values are given in ppm relative to internal $\mathrm{Me}_{4} \mathrm{Si}$. IR spectra were recorded on a JASCO FT/IR-410. MS spectra were taken with a JEOL JMS-DX303 or a JEOL JMS-AX500.
\end{abstract}

1-\{2-(t-Butoxycarbonylamino)ethyl\}-2-triethylsilylethyne 3 . In a two-necked flask equipped with a reflux condenser were placed tetrakis(triphenylphosphine)hydriderhodium ${ }^{10}$ (2 mol\%, $4.6 \mathrm{mg}$ ), 1,1'-bis(diphenylphosphino)ferrocene (dppf) (3 mol\%, $3.3 \mathrm{mg})$, triethylsilylethyne 1 (0.2 mmol, 35.8 $\mu \mathrm{L})$ in acetone $(2.0 \mathrm{~mL})$ under an argon atmosphere, to which bis $\{2$-( $t$-butoxycarbonylamino)ethyl $\}$ disulfide $2^{11}$ (0.6 mmol, $211 \mathrm{mg}$ ) was added. The solution was heated at reflux for $1 \mathrm{~h}$. Then, the solvent was removed under reduced pressure, and the residue was purified by flash column chromatography on silica gel and recycling HPLC using Cadenza CD-C18 (MeOH) giving 3 (48.5 $\mathrm{mg}, 77 \%)$, 2-(t-butoxycarbonylamino)ethanethiol 4 (24.4 mg, 69\%), and 5 (2.5 mg, 4\%). 3: Colorless oil. ${ }^{1} \mathrm{H}-\mathrm{NMR}\left(400 \mathrm{MHz}, \mathrm{CDCl}_{3}\right) \delta 0.60(6 \mathrm{H}, \mathrm{q}, \mathrm{J}=8.0 \mathrm{~Hz}), 0.98(9 \mathrm{H}, \mathrm{t}, \mathrm{J}=8.0 \mathrm{~Hz}), 1.45(9 \mathrm{H}, \mathrm{s})$, $2.82(2 \mathrm{H}, \mathrm{t}, \mathrm{J}=5.6 \mathrm{~Hz}), 3.51(2 \mathrm{H}, \mathrm{q}, \mathrm{J}=5.6 \mathrm{~Hz}), 5.05(1 \mathrm{H}, \mathrm{bs}) .{ }^{13} \mathrm{C}-\mathrm{NMR}\left(100 \mathrm{MHz}, \mathrm{CDCl}_{3}\right) \delta 4.5$, 7.6, 28.4, 35.7, 39.4, 79.5, 93.9, 98.8, 155.5. IR (neat) 3349, 2956, 2875, 2092, 1703, 1507, 1251, 1172, 860, $737 \mathrm{~cm}^{-1}$. MS (EI) m/z $315\left(\mathbf{M}^{+}, 4 \%\right), 230\left(\mathbf{M}^{+}-85,100 \%\right)$. HRMS Calcd for $\mathrm{C}_{15} \mathrm{H}_{29} \mathrm{NO}_{2} \mathrm{SSi}$ : 315.1688. Found: 315.1676. 5: Colorless oil. ${ }^{1} \mathrm{H}-\mathrm{NMR}\left(400 \mathrm{MHz}, \mathrm{CDCl}_{3}\right.$ ) $\delta 0.68$ $(6 \mathrm{H}, \mathrm{q}, \mathrm{J}=7.6 \mathrm{~Hz}), 0.96(9 \mathrm{H}, \mathrm{t}, \mathrm{J}=7.6 \mathrm{~Hz}), 1.44(9 \mathrm{H}, \mathrm{s}), 2.90(2 \mathrm{H}, \mathrm{t}, \mathrm{J}=6.4 \mathrm{~Hz}), 3.36(2 \mathrm{H}, \mathrm{q}, \mathrm{J}=6.4$ $\mathrm{Hz}), 4.83(1 \mathrm{H}, \mathrm{bs}), 5.43(1 \mathrm{H}, \mathrm{s}), 5.50(1 \mathrm{H}, \mathrm{s}) .{ }^{13} \mathrm{C}-\mathrm{NMR}\left(100 \mathrm{MHz}, \mathrm{CDCl}_{3}\right) \delta$ 3.1, 7.4, 28.5, 30.1, 38.8, 79.4, 116.2, 142.0, 155.5. IR (neat) 3354, 2954, 2875, 1697, 1513, 1252, 1172, 1006, $735 \mathrm{~cm}^{-1}$. MS (EI) m/z $317\left(\mathbf{M}^{+}, 12 \%\right), 145\left(\mathbf{M}^{+}-172,100 \%\right)$. HRMS Calcd for $\mathrm{C}_{15} \mathrm{H}_{31} \mathrm{NO}_{2} \mathrm{SSi}$ : 317.1844. Found: 317.1830 .

1-Methylthio-2-triethylsilylethyne. Colorless oil. ${ }^{1} \mathrm{H}-\mathrm{NMR}\left(400 \mathrm{MHz}, \mathrm{CDCl}_{3}\right) \delta 0.60(6 \mathrm{H}, \mathrm{q}, \mathrm{J}=$ $8.0 \mathrm{~Hz}), 0.99(9 \mathrm{H}, \mathrm{t}, \mathrm{J}=7.6 \mathrm{~Hz}), 2.41(3 \mathrm{H}, \mathrm{s}) .{ }^{13} \mathrm{C}-\mathrm{NMR}\left(100 \mathrm{MHz}, \mathrm{CDCl}_{3}\right) \delta$ 4.6, 7.6, 19.7, 96.8, 97.0. IR (neat) 2955, 2932, 2912, 2875, $2092 \mathrm{~cm}^{-1}$. MS (EI) m/z $186\left(\mathbf{M}^{+}, 25 \%\right), 157$ (M+29, 100\%). HRMS Calcd for $\mathrm{C}_{9} \mathrm{H}_{18} \mathrm{SSi}$ : 186.0898. Found: 186.0907. 1-Methylthio-1-triethylsilylethene. Obtained in $2 \%$ yield. ${ }^{1} \mathrm{H}-\mathrm{NMR}\left(400 \mathrm{MHz}, \mathrm{CDCl}_{3}\right) \delta 0.69(6 \mathrm{H}, \mathrm{q}, J=8.0 \mathrm{~Hz}), 0.97(9 \mathrm{H}, \mathrm{t}, J=8.0$ $\mathrm{Hz}), 2.24(3 \mathrm{H}, \mathrm{s}) .{ }^{13} \mathrm{C}-\mathrm{NMR}\left(100 \mathrm{MHz}, \mathrm{CDCl}_{3}\right) \delta 3.2,7.4,13.6,114.5,144.5$. IR (neat) 3064, 2954, 2912, $1738 \mathrm{~cm}^{-1}$. MS (EI) $\mathrm{m} / z .188\left(\mathrm{M}^{+}, 36 \%\right), 159$ (72), 133 (100), 105 (67). HRMS. Calcd for 
$\mathrm{C}_{9} \mathrm{H}_{20} \mathrm{SSi}: 188.1055$. Found: 188.1055 .

1-Butylthio-2-triethylsilylethyne. ${ }^{1} \mathrm{H}-\mathrm{NMR}\left(400 \mathrm{MHz}, \mathrm{CDCl}_{3}\right) \delta 0.60(6 \mathrm{H}, \mathrm{q}, J=8.0 \mathrm{~Hz}), 0.93$ $(3 \mathrm{H}, \mathrm{t}, J=7.6 \mathrm{~Hz}), 0.99(9 \mathrm{H}, \mathrm{t}, J=8.0 \mathrm{~Hz}), 1.45(2 \mathrm{H}$, sixtet, $J=7.6 \mathrm{~Hz}), 1.74(2 \mathrm{H}$, quintet, $J=7.6$ $\mathrm{Hz}), 2.73(2 \mathrm{H}, \mathrm{t}, J=7.6 \mathrm{~Hz}) .{ }^{13} \mathrm{C}-\mathrm{NMR}\left(100 \mathrm{MHz} \mathrm{CDCl}_{3}\right) \delta$ 4.7, 7.6, 13.7, 21.5, 31.2, 35.5, 95.6, 97.8. IR (neat) 2956, 2934, 2913, 2874, $2090 \mathrm{~cm}^{-1}$. MS (EI) m/z $228\left(\mathrm{M}^{+}, 18 \%\right), 199$ (100), 171 (49). HRMS. Calcd for $\mathrm{C}_{12} \mathrm{H}_{26} \mathrm{SSi}$ : 228.1368. Found: 228.1368. 1-Butylthio-1-triethylsilylethene. Obtained in $5 \%$ yield. ${ }^{1} \mathrm{H}-\mathrm{NMR}\left(400 \mathrm{MHz}, \mathrm{CDCl}_{3}\right) \delta 0.68(6 \mathrm{H}, \mathrm{q}, J=8.0 \mathrm{~Hz}), 0.93(3 \mathrm{H}, \mathrm{t}, J=7.2$ $\mathrm{Hz}), 0.96(9 \mathrm{H}, \mathrm{t}, J=8.0 \mathrm{~Hz}), 1.44(2 \mathrm{H}$, sixtet, $J=7.2 \mathrm{~Hz}), 1.63(2 \mathrm{H}$, quintet, $J=7.2 \mathrm{~Hz}), 2.72(2 \mathrm{H}, \mathrm{t}, J$

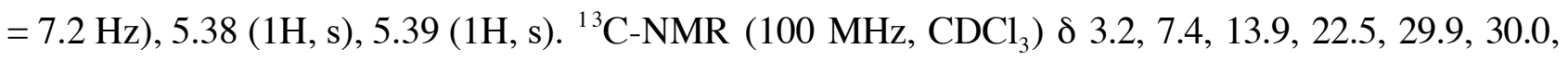
115.1, 143.8. IR (neat) 2955, 2912, 2874, $1551 \mathrm{~cm}^{-1} . \mathrm{MS}$ (EI) $m / z, 230\left(\mathrm{M}^{+}, 44 \%\right), 201$ (67), 175 (98), 145 (100), 115 (98). HRMS. Calcd for $\mathrm{C}_{12} \mathrm{H}_{26} \mathrm{SSi}$ : 230.1524. Found: 230.1524.

1-(3-Methylbutylthio)-2-triethylsilylethyne. Colorless oil. ${ }^{1} \mathrm{H}-\mathrm{NMR}\left(400 \mathrm{MHz}, \mathrm{CDCl}_{3}\right) \delta 0.60$ $(6 \mathrm{H}, \mathrm{q}, \mathrm{J}=8.0 \mathrm{~Hz}), 0.92(6 \mathrm{H}, \mathrm{d}, \mathrm{J}=6.8 \mathrm{~Hz}), 0.99(9 \mathrm{H}, \mathrm{t}, \mathrm{J}=8.0 \mathrm{~Hz}), 1.64(2 \mathrm{H}, \mathrm{q}, \mathrm{J}=7.2 \mathrm{~Hz}), 1.74$ $(1 \mathrm{H}$, septet, $\mathrm{J}=7.2 \mathrm{~Hz}), 2.73(2 \mathrm{H}, \mathrm{t}, \mathrm{J}=7.6 \mathrm{~Hz}) .{ }^{13} \mathrm{C}-\mathrm{NMR}\left(100 \mathrm{MHz}, \mathrm{CDCl}_{3}\right) \delta$ 4.6, 7.6, 22.4, 27.0, 33.9, 38.1, 95.6, 98.0. IR (neat) 2956, 2874, 2097, 1466, $1017 \mathrm{~cm}^{-1}$. MS (EI) m/z $242\left(\mathbf{M}^{+}, 17 \%\right), 213$ ( $\left.\mathbf{M}^{+}-29,100 \%\right)$. HRMS Calcd for $\mathrm{C}_{13} \mathrm{H}_{26} \mathrm{SSi}$ : 242.1524. Found: 242.1531.

1-Octylthio-2-triethylsilylethyne. Colorless oil. ${ }^{1} \mathrm{H}-\mathrm{NMR}\left(400 \mathrm{MHz}, \mathrm{CDCl}_{3}\right) \delta 0.60(6 \mathrm{H}, \mathrm{q}, \mathrm{J}=$ $8.0 \mathrm{~Hz}), 0.88(3 \mathrm{H}, \mathrm{t}, \mathrm{J}=7.6 \mathrm{~Hz}), 0.99(9 \mathrm{H}, \mathrm{t}, \mathrm{J}=8.0 \mathrm{~Hz}), 1.24-1.32(8 \mathrm{H}, \mathrm{m}), 1.38-1.45(2 \mathrm{H}, \mathrm{m}), 1.75$ $(2 \mathrm{H}$, quintet, $\mathrm{J}=7.6 \mathrm{~Hz}), 2.71(2 \mathrm{H}, \mathrm{t}, \mathrm{J}=7.6 \mathrm{~Hz}) .{ }^{13} \mathrm{C}-\mathrm{NMR}\left(100 \mathrm{MHz}, \mathrm{CDCl}_{3}\right) \delta$ 4.6, 7.6, 14.2, 22.8, 28.3, 29.1, 29.20, 29.23, 31.9, 35.8, 95.6, 97.9. IR (neat) 2928, 2855, 2091, 1458, 1017, 863, $725 \mathrm{~cm}^{-1}$. MS (EI) m/z $284\left(\mathbf{M}^{+}, 8 \%\right), 255\left(\mathbf{M}^{+}-29,100 \%\right)$. HRMS Calcd for $\mathrm{C}_{16} \mathrm{H}_{32} \mathrm{SSi}: 284.1994$. Found: 284.1996. 1-Octylthio-1-triethylsilylethene. Obtained in 3\% yield. Colorless oil. ${ }^{1} \mathrm{H}-\mathrm{NMR}$ (400 $\left.\mathrm{MHz}, \mathrm{CDCl}_{3}\right) \delta 0.68(6 \mathrm{H}, \mathrm{q}, \mathrm{J}=7.6 \mathrm{~Hz}), 0.88(3 \mathrm{H}, \mathrm{t}, \mathrm{J}=7.2 \mathrm{~Hz}), 0.96(9 \mathrm{H}, \mathrm{t}, \mathrm{J}=7.2 \mathrm{~Hz}), 1.25-1.35$ $(8 \mathrm{H}, \mathrm{m}), 1.35-1.45(2 \mathrm{H}, \mathrm{m}), 1.63(2 \mathrm{H}$, quintet, $\mathrm{J}=7.2 \mathrm{~Hz}), 2.71(2 \mathrm{H}, \mathrm{t}, \mathrm{J}=7.2 \mathrm{~Hz}), 5.38(2 \mathrm{H}, \mathrm{s}) .{ }^{13} \mathrm{C}-$ NMR (100 MHz, $\left.\mathrm{CDCl}_{3}\right) \delta 3.1,7.4,14.3,22.8,27.9,29.28,29.32,29.4,30.1,31.9,115.1,143.8$. IR (neat) 2926, 2873, 1458, 1247, $1171 \mathrm{~cm}^{-1}$. MS (EI) m/z $286\left(\mathbf{M}^{+}, 24 \%\right), 145\left(\mathbf{M}^{+}-141,100 \%\right)$. HRMS Calcd for $\mathrm{C}_{16} \mathrm{H}_{34} \mathrm{SSi}: 286.2150$. Found: 286.2136 .

1-(2-Methoxyethylthio)-2-triethylsilylethyne. Colorless oil. ${ }^{1} \mathrm{H}-\mathrm{NMR}\left(400 \mathrm{MHz}, \mathrm{CDCl}_{3}\right) \delta 0.60$ $(6 \mathrm{H}, \mathrm{q}, \mathrm{J}=8.0 \mathrm{~Hz}), 0.99(9 \mathrm{H}, \mathrm{t}, \mathrm{J}=8.0 \mathrm{~Hz}), 2.89(2 \mathrm{H}, \mathrm{t}, \mathrm{J}=6.8 \mathrm{~Hz}), 3.39(3 \mathrm{H}, \mathrm{s}), 3.70(2 \mathrm{H}, \mathrm{t}, \mathrm{J}=6.8$ $\mathrm{Hz}) .{ }^{13} \mathrm{C}-\mathrm{NMR}\left(100 \mathrm{MHz}, \mathrm{CDCl}_{3}\right) \delta 4.6,7.6,34.9,58.9,70.2,94.5,98.3$. IR (neat) 2955, 2874, 2091, 1457, 1119, 860, $727 \mathrm{~cm}^{-1}$. MS (EI) m/z $230\left(\mathbf{M}^{+}, 36 \%\right), 201\left(\mathbf{M}^{+}-29,100 \%\right)$. HRMS Calcd for $\mathrm{C}_{11} \mathrm{H}_{22}$ OSSi: 230.1161. Found: 230.1161 .

1-(2-Methoxycarbonylethylthio)-2-triethylsilylethyne. Colorless oil. ${ }^{1} \mathrm{H}-\mathrm{NMR}$ (400 MHz, $\left.\mathrm{CDCl}_{3}\right) \delta 0.60(6 \mathrm{H}, \mathrm{q}, \mathrm{J}=8.0 \mathrm{~Hz}), 0.99(9 \mathrm{H}, \mathrm{t}, \mathrm{J}=8.0 \mathrm{~Hz}), 2.81(2 \mathrm{H}, \mathrm{t}, \mathrm{J}=7.2 \mathrm{~Hz}), 2.95(2 \mathrm{H}, \mathrm{t}, \mathrm{J}=$ $7.2 \mathrm{~Hz}), 3.71(3 \mathrm{H}, \mathrm{s}) .{ }^{13} \mathrm{C}-\mathrm{NMR}\left(100 \mathrm{MHz}, \mathrm{CDCl}_{3}\right) \delta$ 4.6, 7.6, 30.4, 33.8, 52.0, 94.0, 99.5, 171.6. IR (neat) 2955, 2091, 1743, 1245, 1017, 859, $737 \mathrm{~cm}^{-1}$. MS (EI) m/z $258\left(\mathbf{M}^{+}, 29 \%\right), 201\left(\mathbf{M}^{+}-57,100 \%\right)$. HRMS Calcd for $\mathrm{C}_{12} \mathrm{H}_{22} \mathrm{O}_{2}$ SSi: 258.1110. Found: 258.1103. 1-(2-Methoxycarbonylethylthio)-1triethylsilylethene. Obtained in $2 \%$ yield. Colorless oil. ${ }^{1} \mathrm{H}-\mathrm{NMR}\left(400 \mathrm{MHz}, \mathrm{CDCl}_{3}\right) \delta 0.67(6 \mathrm{H}$, $\mathrm{q}, \mathrm{J}=8.0 \mathrm{~Hz}), 0.95(9 \mathrm{H}, \mathrm{t}, \mathrm{J}=8.0 \mathrm{~Hz}), 2.66(2 \mathrm{H}, \mathrm{t}, \mathrm{J}=7.2 \mathrm{~Hz}), 3.02(2 \mathrm{H}, \mathrm{t}, \mathrm{J}=7.6 \mathrm{~Hz}), 3.70(3 \mathrm{H}, \mathrm{s})$, 
$5.42(1 \mathrm{H}, \mathrm{s}), 5.43(1 \mathrm{H}, \mathrm{s}) .{ }^{13} \mathrm{C}-\mathrm{NMR}\left(100 \mathrm{MHz}, \mathrm{CDCl}_{3}\right) \delta 3.1,7.4,24.8,32.8,51.9,115.8,128.3$, 176.9. IR (neat) 2953, 2875, 1742, 1437, 1241, 1007, $722 \mathrm{~cm}^{-1} . \mathrm{MS}$ (EI) m/z $260\left(\mathbf{M}^{+}, 17 \%\right), 231$ (M+29, 100\%). HRMS Calcd for $\mathrm{C}_{12} \mathrm{H}_{24} \mathrm{O}_{2} \mathrm{SSi}$ : 260.1266. Found: 260.1266.

1-Methylthio-2-triisopropylsilylethyne. $\mathrm{RhH}\left(\mathrm{PPh}_{3}\right)_{4}(4 \mathrm{~mol} \%)$ and dppf (6 mol\%) were used, which improved the yield of the product. Colorless oil. ${ }^{1} \mathrm{H}-\mathrm{NMR}\left(400 \mathrm{MHz}, \mathrm{CDCl}_{3}\right) \delta 2.41(3 \mathrm{H}, \mathrm{s})$, $1.06(21 \mathrm{H}, \mathrm{s}) .{ }^{13} \mathrm{C}-\mathrm{NMR}\left(100 \mathrm{MHz}, \mathrm{CDCl}_{3}\right) \delta 11.5,18.7,19.9,95.8,97.4$. IR (neat) 2943, 2892, 2866, 2091, $1462 \mathrm{~cm}^{-1}$. MS (EI) m/z $228\left(\mathbf{M}^{+}, 7 \%\right), 185\left(\mathbf{M}^{+}-43,100 \%\right)$. HRMS Calcd for $\mathrm{C}_{12} \mathrm{H}_{24} \mathrm{SSi}$ : 228.1368. Found: 228.1860.

1-Octylthio-2-triisopropylsilylethyne. Colorless oil. ${ }^{1} \mathrm{H}-\mathrm{NMR}\left(400 \mathrm{MHz}, \mathrm{CDCl}_{3}\right) \delta 0.88(3 \mathrm{H}, \mathrm{t}, \mathrm{J}$ $=6.8 \mathrm{~Hz}), 1.07(21 \mathrm{H}, \mathrm{s}), 1.24-1.32(8 \mathrm{H}, \mathrm{m}), 1.38-1.43(2 \mathrm{H}, \mathrm{m}), 1.77(2 \mathrm{H}$, quintet, J = 7.2 Hz), 2.71 $(2 \mathrm{H}, \mathrm{t}, \mathrm{J}=7.2 \mathrm{~Hz}) .{ }^{13} \mathrm{C}-\mathrm{NMR}\left(100 \mathrm{MHz}, \mathrm{CDCl}_{3}\right) \delta 11.5,14.2,18.7,22.8,28.3,29.19,29.24,31.9$, 35.9, 95.9, 96.8. IR (neat) 2927, 2865, 2089, 1463, 995, $882 \mathrm{~cm}^{-1}$. MS (EI) m/z $326\left(\mathbf{M}^{+}, 12 \%\right), 283$ $\left(\mathbf{M}^{+}-43,100 \%\right)$. HRMS Calcd for $\mathrm{C}_{19} \mathrm{H}_{38} \mathrm{SSi}$ : 326.2463. Found: 326.2446.

1-Octylthio-2-(2,6-dimethylphenyl)ethyne. Colorless oil. ${ }^{1} \mathrm{H}-\mathrm{NMR}\left(400 \mathrm{MHz}, \mathrm{CDCl}_{3}\right) \delta 0.88$ $(3 \mathrm{H}, \mathrm{t}, \mathrm{J}=7.2 \mathrm{~Hz}), 1.24-1.34(8 \mathrm{H}, \mathrm{m}), 1.42-1.50(2 \mathrm{H}, \mathrm{m}), 1.83(2 \mathrm{H}$, quintet, J = 7.2 Hz), $2.40(6 \mathrm{H}, \mathrm{s})$, $2.80(2 \mathrm{H}, \mathrm{t}, \mathrm{J}=7.2 \mathrm{~Hz}), 7.00(2 \mathrm{H}, \mathrm{d}, \mathrm{J}=7.2 \mathrm{~Hz}), 7.06(1 \mathrm{H}, \mathrm{dd}, \mathrm{J}=8.8,6.4 \mathrm{~Hz}) .{ }^{13} \mathrm{C}-\mathrm{NMR}(100 \mathrm{MHz}$, $\left.\mathrm{CDCl}_{3}\right) \delta 14.2,21.2,22.8,28.4,29.2,29.3,29.5,31.9,36.3,87.4,90.7,123.3,126.5,127.1,139.8$. IR (neat) 2926, 2854, 2158, 1465, $768 \mathrm{~cm}^{-1}$. MS (EI) m/z $274\left(\mathbf{M}^{+}, 100 \%\right), 162\left(\mathbf{M}^{+}-112,73 \%\right)$. HRMS Calcd for $\mathrm{C}_{18} \mathrm{H}_{26} \mathrm{~S}$ : 274.1755. Found: 274.1740. 1-Octylthio-1-(2,6-dimethylphenyl)ethene. Obtained in $38 \%$ yield. Colorless oil. ${ }^{1} \mathrm{H}-\mathrm{NMR}\left(400 \mathrm{MHz}, \mathrm{CDCl}_{3}\right) \delta 0.88(3 \mathrm{H}, \mathrm{t}, \mathrm{J}=6.8 \mathrm{~Hz}), 1.24-$ $1.32(8 \mathrm{H}, \mathrm{m}), 1.37-1.45(2 \mathrm{H}, \mathrm{m}), 1.67(2 \mathrm{H}$, quintet, $\mathrm{J}=7.2 \mathrm{~Hz}), 2.33(6 \mathrm{H}, \mathrm{s}), 2.70(2 \mathrm{H}, \mathrm{t}, \mathrm{J}=7.2 \mathrm{~Hz})$, $4.99(1 \mathrm{H}, \mathrm{s}), 5.14(1 \mathrm{H}, \mathrm{s}), 7.01(2 \mathrm{H}, \mathrm{d}, \mathrm{J}=7.2 \mathrm{~Hz}), 7.08(1 \mathrm{H}, \mathrm{dd}, \mathrm{J}=8.0,6.4 \mathrm{~Hz}) .{ }^{13} \mathrm{C}-\mathrm{NMR}(100$ $\left.\mathrm{MHz}, \mathrm{CDCl}_{3}\right) \delta 14.2,19.7,22.8,28.2,29.3,31.7,31.9,107.7,127.1,127.4,136.1,139.2,143.9$. IR (neat) 2925, 2854, 1593, 1465, 1064, 849, $768 \mathrm{~cm}^{-1}$. MS (EI) m/z $276\left(\mathbf{M}^{+}, 27 \%\right), 163\left(\mathbf{M}^{+}-113\right.$, 100\%). HRMS Calcd for $\mathrm{C}_{18} \mathrm{H}_{28} \mathrm{~S}: 276.1912$. Found: 276.1901.

1-Methylthio-2-(2,4,6-trimethylphenyl)ethyne. Colorless oil. ${ }^{1} \mathrm{H}-\mathrm{NMR}$ (400 $\mathrm{MHz}, \mathrm{CDCl}_{3}$ )

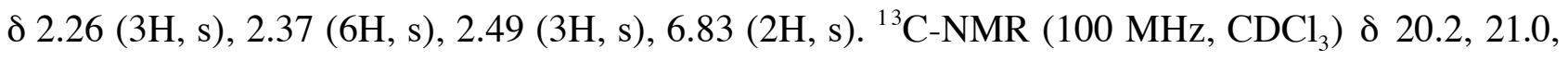
21.4, 87.6, 89.8, 120.1, 127.4, 137.2, 139.9. IR (neat) 2924, 2854, 2732, 2158, $1609 \mathrm{~cm}^{-1}$. MS (EI) m/z $190\left(\mathbf{M}^{+}, 100 \%\right), 143\left(\mathbf{M}^{+}-47,91 \%\right)$. HRMS Calcd for $\mathrm{C}_{12} \mathrm{H}_{14} \mathrm{~S}: 190.0816$. Found: 190.0803. 1Methylthio-1-(2,4,6-trimethylphenyl)ethene. Obtained in $18 \%$ yield. ${ }^{1} \mathrm{H}-\mathrm{NMR}$ (400 $\mathrm{MHz}$, $\left.\mathrm{CDCl}_{3}\right)$ \& $2.25(3 \mathrm{H}, \mathrm{s}), 2.27(3 \mathrm{H}, \mathrm{s}), 2.28(6 \mathrm{H}, \mathrm{s}), 5.00(1 \mathrm{H}, \mathrm{s}), 5.09(1 \mathrm{H}, \mathrm{s}), 6.85(2 \mathrm{H}, \mathrm{s}) .{ }^{13} \mathrm{C}-\mathrm{NMR}$ $\left(100 \mathrm{MHz}, \mathrm{CDCl}_{3}\right) \delta 15.1,19.6,21.1,107.4,127.9,136.0,136.1,137.0,144.9$. IR (neat) 3090, 2198, 2856, $1602 \mathrm{~cm}^{-1}$. MS (EI) $\mathrm{m} / z 192\left(\mathrm{M}^{+}, 67 \%\right), 177$ (100). HRMS. Calcd for $\mathrm{C}_{12} \mathrm{H}_{16} \mathrm{~S}: 192.0973$. Found: 192.0999 .

1-Methylthio-2-(1-adamantyl)ethyne. ${ }^{1} \mathrm{H}-\mathrm{NMR}\left(400 \mathrm{MHz}, \mathrm{CDCl}_{3}\right) \delta 1.67(6 \mathrm{H}, \mathrm{t}, J=2.8 \mathrm{~Hz})$, $1.85(6 \mathrm{H}, \mathrm{d}, J=2.8 \mathrm{~Hz}), 1.92-1.98(3 \mathrm{H}, \mathrm{br}), 2.34(3 \mathrm{H}, \mathrm{s}) .{ }^{13} \mathrm{C}-\mathrm{NMR}\left(100 \mathrm{MHz}, \mathrm{CDCl}_{3}\right) \delta 19.8$, 28.1, 30.9, 36.4, 42.9, 68.9, 100.9. IR (neat) 2906, 2849, $1450 \mathrm{~cm}^{-1} . \mathrm{MS}(\mathrm{EI}) \mathrm{m} / z .206\left(\mathrm{M}^{+}, 100 \%\right), 191$ (82). HRMS. Calcd for $\mathrm{C}_{13} \mathrm{H}_{18} \mathrm{~S}$ : 206.1129. Found: 206.1102. Mp. 67-69 ${ }^{\circ} \mathrm{C}$ (hexane). 1Methylthio-1-(1-adamantyl)ethene. Obtained in $12 \%$ yield. ${ }^{1} \mathrm{H}-\mathrm{NMR}\left(400 \mathrm{MHz}, \mathrm{CDCl}_{3}\right) \delta 1.68$ 
$(3 \mathrm{H}, \mathrm{d}, J=11.6 \mathrm{~Hz}), 1.73(3 \mathrm{H}, \mathrm{d}, J=12.0 \mathrm{~Hz}), 1.83(6 \mathrm{H}, \mathrm{d}, J=3.2 \mathrm{~Hz}), 1.99-2.06(3 \mathrm{H}, \mathrm{br}), 2.20$ $(3 \mathrm{H}, \mathrm{s}) .{ }^{13} \mathrm{C}-\mathrm{NMR}\left(100 \mathrm{MHz}, \mathrm{CDCl}_{3}\right) \delta 14.8,28.9,36.9,39.3,42.2,100.7,158.0$. IR (neat) 2900, 2847, $1588 \mathrm{~cm}^{-1}$. MS (EI) $m / z 208\left(\mathrm{M}^{+}, 100 \%\right), 135$ (38). HRMS. Calcd for $\mathrm{C}_{13} \mathrm{H}_{20} \mathrm{~S}: 208.1286$. Found: 208.1251. Mp. $62-64{ }^{\circ} \mathrm{C}$ (hexane).

1-Octylthio-2-(1-adamantyl)ethyne. Colorless oil. ${ }^{1} \mathrm{H}-\mathrm{NMR}\left(400 \mathrm{MHz}, \mathrm{CDCl}_{3}\right) \delta 0.88(3 \mathrm{H}, \mathrm{t}, \mathrm{J}=$ $6.8 \mathrm{~Hz}), 1.24-1.33(8 \mathrm{H}, \mathrm{m}), 1.37-1.44(2 \mathrm{H}, \mathrm{m}), 1.67(6 \mathrm{H}, \mathrm{bs}), 1.71(2 \mathrm{H}$, quintet, $\mathrm{J}=7.2 \mathrm{~Hz}), 1.86(6 \mathrm{H}$, bd, J = 3.2 Hz), 1.94 (3H, bs), $2.64(2 \mathrm{H}, \mathrm{t}, \mathrm{J}=7.2 \mathrm{~Hz}) \cdot{ }^{13} \mathrm{C}-\mathrm{NMR}\left(100 \mathrm{MHz}, \mathrm{CDCl}_{3}\right) \delta 14.3,22.8$, 28.1, 28.3, 29.1, 29.2, 29.3, 31.0, 31.9, 35.7, 36.4, 43.0,67.3, 102.1. IR (neat) 2925, 2851, 2657, 1451 $\mathrm{cm}^{-1}$. MS (EI) m/z $304\left(\mathbf{M}^{+}, 84 \%\right), 135\left(\mathbf{M}^{+}-169,100 \%\right)$. HRMS Calcd for $\mathrm{C}_{20} \mathrm{H}_{32} \mathrm{~S}: 304.2225$. Found: 304.2214. 1-Octylthio-1-(1-adamantyl)ethene. Obtained in 7\% yield. Colorless oil. ${ }^{1} \mathrm{H}-$ NMR (400 MHz, $\left.\mathrm{CDCl}_{3}\right) \delta 0.88(3 \mathrm{H}, \mathrm{t}, \mathrm{J}=7.2 \mathrm{~Hz}), 1.21-1.33(8 \mathrm{H}, \mathrm{m}), 1.35-1.45(2 \mathrm{H}, \mathrm{m}), 1.64(2 \mathrm{H}$, quintet, $\mathrm{J}=7.6 \mathrm{~Hz}), 1.69(6 \mathrm{H}, \mathrm{bd}, \mathrm{J}=8.0 \mathrm{~Hz}), 1.82(6 \mathrm{H}, \mathrm{d}, \mathrm{J}=2.4 \mathrm{~Hz}), 2.01(3 \mathrm{H}, \mathrm{bs}), 2.65(2 \mathrm{H}, \mathrm{t}, \mathrm{J}=$ $7.2 \mathrm{~Hz}), 4.64(1 \mathrm{H}, \mathrm{s}), 5.07(1 \mathrm{H}, \mathrm{s}) .{ }^{13} \mathrm{C}-\mathrm{NMR}\left(100 \mathrm{MHz}, \mathrm{CDCl}_{3}\right) \delta$ 14.3, 22.8, 28.2, 28.9, 29.29, $29.34,29.4,31.9,36.9,39.5,42.1,102.2,156.9$. IR (neat) $2924,2850,1590,1451 \mathrm{~cm}^{-1}$. MS (EI) m/z $306\left(\mathbf{M}^{+}, 17 \%\right), 194\left(\mathbf{M}^{+}-112,100 \%\right)$. HRMS Calcd for $\mathrm{C}_{20} \mathrm{H}_{34} \mathrm{~S}: 306.2381$. Found: 306.2385.

1-Phenylthio-2-triethylsilylethyne. In a two-necked flask were placed tetrakis(triphenylphosphine)hydriderhodium ( $2 \mathrm{~mol} \%, 4.6 \mathrm{mg}$ ) and dppf (4 mol\%, $3.3 \mathrm{mg}$ ) in acetone $(1.5 \mathrm{~mL})$ under an argon atmosphere, and the mixture was heated at reflux for $10 \mathrm{~min}$. After cooled to room temperature, $\mathbf{1}(1.0 \mathrm{mmol}, 179 \mu \mathrm{L})$ and diphenyl disulfide $(0.2 \mathrm{mmol}, 43.6 \mathrm{mg})$ were added, and the solution was stirred at the temperature for $12 \mathrm{~h}$. Then, the solvent was removed under reduced pressure, and the residue was purified by flash column chromatography on silica gel and recycling HPLC using Cadenza CD-C18 (MeOH) giving the title compound (42.7 mg, 86\%) and 1-phenylthio1-triethylsilylethene (20 mg, 40\%). Colorless oil. ${ }^{1} \mathrm{H}-\mathrm{NMR}\left(400 \mathrm{MHz}, \mathrm{CDCl}_{3}\right) \delta 0.68(6 \mathrm{H}, \mathrm{q}, \mathrm{J}=8.0$ $\mathrm{Hz}), 1.04(9 \mathrm{H}, \mathrm{t}, \mathrm{J}=8.0 \mathrm{~Hz}), 7.20(1 \mathrm{H}, \mathrm{t}, \mathrm{J}=7.6 \mathrm{~Hz}), 7.32(2 \mathrm{H}, \mathrm{t}, \mathrm{J}=7.6 \mathrm{~Hz}), 7.41(2 \mathrm{H}, \mathrm{d}, \mathrm{J}=8.0$ $\mathrm{Hz}) .{ }^{13} \mathrm{C}-\mathrm{NMR}\left(100 \mathrm{MHz}, \mathrm{CDCl}_{3}\right) \delta 4.6,7.6,90.7,104.1,125.9,126.3,129.0,132.4$. IR (neat) 2955, 2874, 2094, 1478, 1018, $862 \mathrm{~cm}^{-1}$. MS (EI) m/z $248\left(\mathbf{M}^{+}, 36 \%\right), 219\left(\mathbf{M}^{+}-29,100 \%\right)$. HRMS Calcd for $\mathrm{C}_{14} \mathrm{H}_{20} \mathrm{OSSi}$ : 248.1055. Found: 248.1031. 1-Phenylthio-1-triethylsilylethene. Colorless oil. ${ }^{1} \mathrm{H}-\mathrm{NMR}\left(400 \mathrm{MHz}, \mathrm{CDCl}_{3}\right) \delta 0.72(6 \mathrm{H}, \mathrm{q}, \mathrm{J}=8.0 \mathrm{~Hz}), 1.00(9 \mathrm{H}, \mathrm{t}, \mathrm{J}=8.0 \mathrm{~Hz}), 5.20(1 \mathrm{H}, \mathrm{s}), 5.42$ $(1 \mathrm{H}, \mathrm{s}), 7.30-7.37(3 \mathrm{H}, \mathrm{m}), 7.44(2 \mathrm{H}, \mathrm{d}, \mathrm{J}=7.2 \mathrm{~Hz}) .{ }^{13} \mathrm{C}-\mathrm{NMR}\left(100 \mathrm{MHz}, \mathrm{CDCl}_{3}\right) \delta 3.3,7.4,120.1$, 128.1, 129.0, 131.9, 134.8, 145.0. IR (neat) 2953, 2874, 1477, 1238, 1006, $777 \mathrm{~cm}^{-1}$. MS (EI) m/z 250 $\left(\mathbf{M}^{+}, 48 \%\right), 195$ (M+55, 100\%). HRMS Calcd for $\mathrm{C}_{14} \mathrm{H}_{22} \mathrm{SSi}: 250.1211$. Found: 250.1172 .

1-(p-Tolylthio)-2-triethylsilylethyne. Colorless oil. ${ }^{1} \mathrm{H}-\mathrm{NMR}\left(400 \mathrm{MHz}, \mathrm{CDCl}_{3}\right) \delta 0.66(6 \mathrm{H}, \mathrm{q}, \mathrm{J}$ $=8.0 \mathrm{~Hz}), 1.03(9 \mathrm{H}, \mathrm{t}, \mathrm{J}=8.0 \mathrm{~Hz}), 2.32(3 \mathrm{H}, \mathrm{s}), 7.13(2 \mathrm{H}, \mathrm{d}, \mathrm{J}=8.0 \mathrm{~Hz}), 7.30(2 \mathrm{H}, \mathrm{d}, \mathrm{J}=8.8 \mathrm{~Hz})$. ${ }^{13} \mathrm{C}-\mathrm{NMR}\left(100 \mathrm{MHz}, \mathrm{CDCl}_{3}\right) \delta$ 4.6, 7.6, 21.1, 91.4, 103.4, 126.2, 128.7, 129.8, 136.3. IR (neat) 2955, 2874, 2094, 1492, 1016, 862, $802 \mathrm{~cm}^{-1}$. MS (EI) m/z $262\left(\mathbf{M}^{+}, 60 \%\right), 233\left(\mathbf{M}^{+}-29,100 \%\right)$. HRMS Calcd for $\mathrm{C}_{15} \mathrm{H}_{22} \mathrm{SSi}$ : 262.1211. Found: 262.1181. 1-(p-Tolylthio)-1-triethylsilylethene. Obtained in $28 \%$ yield. Colorless oil. ${ }^{1} \mathrm{H}-\mathrm{NMR}\left(400 \mathrm{MHz}, \mathrm{CDCl}_{3}\right) \delta 0.71(6 \mathrm{H}, \mathrm{q}, \mathrm{J}=8.0 \mathrm{~Hz}), 1.00$ $(9 \mathrm{H}, \mathrm{t}, \mathrm{J}=8.0 \mathrm{~Hz}), 2.36(3 \mathrm{H}, \mathrm{s}), 5.13(1 \mathrm{H}, \mathrm{s}), 5.37(1 \mathrm{H}, \mathrm{s}), 7.16(2 \mathrm{H}, \mathrm{d}, \mathrm{J}=8.0 \mathrm{~Hz}), 7.32(2 \mathrm{H}, \mathrm{d}, \mathrm{J}=$ $8.0 \mathrm{~Hz}) .{ }^{13} \mathrm{C}-\mathrm{NMR}\left(100 \mathrm{MHz}, \mathrm{CDCl}_{3}\right) \delta 3.3,7.4,21.4,119.0,130.0,131.5,135.0,138.3,145.4$. IR (neat) 2953, 2874, 1491, 1458, 1238, $1005 \mathrm{~cm}^{-1} . \mathrm{MS}$ (EI) m/z $264\left(\mathbf{M}^{+}, 75 \%\right), 209\left(\mathbf{M}^{+}-55,100 \%\right)$. 
HRMS Calcd for $\mathrm{C}_{15} \mathrm{H}_{24} \mathrm{SSi}$ : 264.1368. Found: 264.1338.

1-(p-Chlorophenyl)thio-2-triethylsilylethyne. Colorless oil. ${ }^{1} \mathrm{H}-\mathrm{NMR}\left(400 \mathrm{MHz}, \mathrm{CDCl}_{3}\right) \delta 0.67$ $(6 \mathrm{H}, \mathrm{q}, \mathrm{J}=8.0 \mathrm{~Hz}), 1.03(9 \mathrm{H}, \mathrm{t}, \mathrm{J}=8.0 \mathrm{~Hz}), 7.29(2 \mathrm{H}, \mathrm{d}, \mathrm{J}=8.8 \mathrm{~Hz}), 7.34(2 \mathrm{H}, \mathrm{d}, \mathrm{J}=8.8 \mathrm{~Hz}) .{ }^{13} \mathrm{C}-$ NMR $\left(100 \mathrm{MHz}, \mathrm{CDCl}_{3}\right) \delta 4.6,7.6,90.1,104.8,127.2,129.2,131.1,132.3$. IR (neat) 2955, 2874, 2095, 1475, 1089, 1011, $861 \mathrm{~cm}^{-1}$. MS (EI) m/z $282\left(\mathbf{M}^{+}, 41 \%\right), 253\left(\mathbf{M}^{+}-29,100 \%\right)$. HRMS Calcd for $\mathrm{C}_{14} \mathrm{H}_{19} \mathrm{SSiCl}$ : 282.0665. Found: 282.0661. 1-(p-Chlorophenyl)thio-1-triethylsilylethene. Obtained in $19 \%$ yield. Colorless oil. ${ }^{1} \mathrm{H}-\mathrm{NMR}\left(400 \mathrm{MHz}, \mathrm{CDCl}_{3}\right) \delta 0.71(6 \mathrm{H}, \mathrm{q}, \mathrm{J}=8.0 \mathrm{~Hz}), 0.99$ $(9 \mathrm{H}, \mathrm{t}, \mathrm{J}=8.0 \mathrm{~Hz}), 5.21(1 \mathrm{H}, \mathrm{s}), 5.45(1 \mathrm{H}, \mathrm{s}), 7.31(2 \mathrm{H}, \mathrm{d}, \mathrm{J}=8.8 \mathrm{~Hz}), 7.36(2 \mathrm{H}, \mathrm{d}, \mathrm{J}=8.8 \mathrm{~Hz}) .{ }^{13} \mathrm{C}-$ NMR (100 MHz, $\left.\mathrm{CDCl}_{3}\right) \delta 3.2,7.4,120.7,129.3,130.5,132.2,135.9,138.1$. IR (neat) 2954, 2875, 1474, 1093, 1014, $822 \mathrm{~cm}^{-1}$. MS (EI) m/z $284\left(\mathbf{M}^{+}, 52 \%\right), 229\left(\mathbf{M}^{+}-55,100 \%\right)$. HRMS Calcd for $\mathrm{C}_{14} \mathrm{H}_{21} \mathrm{SSiCl}$ : 284.0822. Found: 284.0809 .

1-Phenylthio-2-(2,4,6-trimethylphenyl)ethyne. Colorless oil. ${ }^{1} \mathrm{H}-\mathrm{NMR}$ (400 $\left.\mathrm{MHz}, \mathrm{CDCl}_{3}\right)$

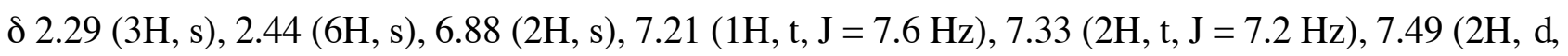
$\mathrm{J}=7.2 \mathrm{~Hz}) .{ }^{13} \mathrm{C}-\mathrm{NMR}\left(100 \mathrm{MHz}, \mathrm{CDCl}_{3}\right) \delta 21.2,21.5,81.7,95.8,119.7,125.8,126.2,127.6,129.0$, 133.6, 138.1, 140.5. IR (neat) 2916, 2156, 1609, 1582, 1478, 1440, $1024 \mathrm{~cm}^{-1} . \mathrm{MS}$ (EI) m/z $252\left(\mathbf{M}^{+}\right.$, $100 \%), 219$ ( $\left.\mathbf{M}^{+}-33,58 \%\right)$. HRMS Calcd for $\mathrm{C}_{17} \mathrm{H}_{16} \mathrm{~S}: 252.0973$. Found: 252.0945. 1-Phenylthio1-(2,4,6-trimethylphenyl)ethene. Obtained in $46 \%$ yield. Colorless oil. ${ }^{1} \mathrm{H}-\mathrm{NMR}$ (400 MHz, $\left.\mathrm{CDCl}_{3}\right) \delta 2.27(3 \mathrm{H}, \mathrm{s}), 2.37(6 \mathrm{H}, \mathrm{s}), 4.83(1 \mathrm{H}, \mathrm{s}), 4.95(1 \mathrm{H}, \mathrm{s}), 6.86(2 \mathrm{H}, \mathrm{s}), 7.37-7.39$ (3H, m), 7.56$7.58(2 \mathrm{H}, \mathrm{m}) .{ }^{13} \mathrm{C}-\mathrm{NMR}\left(100 \mathrm{MHz}, \mathrm{CDCl}_{3}\right) \delta$ 19.7, 21.2, 109.8, 128.0, 128.8, 129.2, 131.7, 135.5, 136.2, 137.3, 142.4, 145.6. IR (neat) 2918, 1604, 1475, 1439, 1158, $1065 \mathrm{~cm}^{-1}$. MS (EI) m/z $254\left(\mathbf{M}^{+}\right.$, 49\%), $145\left(\mathbf{M}^{+}-109,100 \%\right)$. HRMS Calcd for $\mathrm{C}_{17} \mathrm{H}_{18} \mathrm{~S}: 254.1129$. Found: 254.1121.

1-Phenylthio-2-(1-methoxycyclohexyl)ethyne. $\mathrm{RhH}\left(\mathrm{PPh}_{3}\right)_{4}(2.5 \mathrm{~mol} \%)$ and dppf $(5 \mathrm{~mol} \%)$ were used, and the mixture was heated at reflux for $2 \mathrm{~h}$. Colorless oil. ${ }^{1} \mathrm{H}-\mathrm{NMR}\left(400 \mathrm{MHz}, \mathrm{CDCl}_{3}\right)$

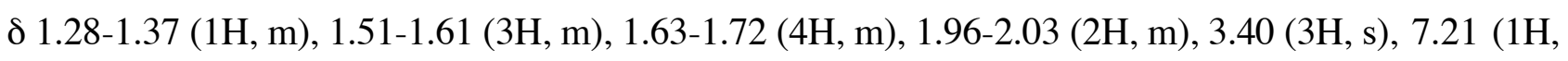
$\mathrm{t}, \mathrm{J}=7.6 \mathrm{~Hz}), 7.33(2 \mathrm{H}, \mathrm{t}, \mathrm{J}=8.0 \mathrm{~Hz}), 7.40(2 \mathrm{H}, \mathrm{d}, \mathrm{J}=7.6 \mathrm{~Hz}) .{ }^{13} \mathrm{C}-\mathrm{NMR}\left(100 \mathrm{MHz}, \mathrm{CDCl}_{3}\right) \delta 22.9$, 25.5, 36.8, 51.0, 71.9, 75.0, 100.4, 125.8, 126.2, 129.1, 132.9. IR (neat) 2935, 2857, 2168, 1583, 1478, 1442, 1285, 1091, $925 \mathrm{~cm}^{-1}$. MS (EI) m/z $246\left(\mathbf{M}^{+}, 62 \%\right), 203\left(\mathbf{M}^{+}-43,100 \%\right)$. HRMS Calcd for $\mathrm{C}_{15} \mathrm{H}_{18} \mathrm{OS}: 246.1078$. Found: 246.1054. 1-Phenylthio-1-(1-methoxycyclohexyl)ethene. Obtained in $88 \%$ yield. Colorless oil. ${ }^{1} \mathrm{H}-\mathrm{NMR}\left(400 \mathrm{MHz}, \mathrm{CDCl}_{3}\right) \delta 1.20-1.29(1 \mathrm{H}, \mathrm{m}), 1.52-1.67(7 \mathrm{H}, \mathrm{m})$, 2.04-2.07 (2H, m), $4.62(1 \mathrm{H}, \mathrm{s}), 5.21(1 \mathrm{H}, \mathrm{s}), 7.31-7.37(3 \mathrm{H}, \mathrm{m}), 7.48(2 \mathrm{H}, \mathrm{d}, \mathrm{J}=7.6 \mathrm{~Hz}) .{ }^{13} \mathrm{C}-\mathrm{NMR}$ $\left(100 \mathrm{MHz} \mathrm{CDCl}_{3}\right) \delta 21.9,25.9,34.2,49.7,78.6,110.1,128.1,129.1,133.0,135.0,153.5$. IR (neat) 2935, 2856, 1602, 1439, 1147, 1074, 930, $871 \mathrm{~cm}^{-1} . \mathrm{MS}(\mathrm{EI}) \mathrm{m} / \mathrm{z} 248\left(\mathbf{M}^{+}, 19 \%\right), 113\left(\mathbf{M}^{+}-135\right.$, 100\%). HRMS Calcd for $\mathrm{C}_{15} \mathrm{H}_{20} \mathrm{OS}: 248.1235$. Found: 248.1217.

1-Phenylthio-2-\{1-(t-butyldimethylsilyloxy)cyclohexyl\}ethyne. $\mathrm{RhH}\left(\mathrm{PPh}_{3}\right)_{4}(2.5 \mathrm{~mol} \%)$ and $\operatorname{dppf}(5 \mathrm{~mol} \%)$ were used, and the mixture was heated at reflux for $2 \mathrm{~h}$. Colorless oil. ${ }^{1} \mathrm{H}-\mathrm{NMR}(400$ $\left.\mathrm{MHz}, \mathrm{CDCl}_{3}\right) \delta 0.16(6 \mathrm{H}, \mathrm{s}), 0.89(9 \mathrm{H}, \mathrm{s}), 1.31-1.39(1 \mathrm{H}, \mathrm{m}), 1.41-1.49(1 \mathrm{H}, \mathrm{m}), 1.50-1.60(2 \mathrm{H}, \mathrm{m})$, 1.64-1.74 (4H, m), 1.85-1.92 (2H, m), $7.20(1 \mathrm{H}, \mathrm{t}, \mathrm{J}=7.2 \mathrm{~Hz}), 7.32(2 \mathrm{H}, \mathrm{t}, \mathrm{J}=7.2 \mathrm{~Hz}), 7.41(2 \mathrm{H}, \mathrm{d}, \mathrm{J}$ $=7.2 \mathrm{~Hz}) .{ }^{13} \mathrm{C}-\mathrm{NMR}\left(100 \mathrm{MHz}, \mathrm{CDCl}_{3}\right) \delta-2.7,18.3,22.9,25.4,26.0,41.1,70.2,70.7,103.8,125.9$, 126.2, 129.0. IR (neat) 2933, 2856, 2173, 1479, 1253, 1095, 1052, $837 \mathrm{~cm}^{-1}$. MS (EI) m/z $346\left(\mathbf{M}^{+}\right.$, 
0.3\%), 289 ( $\left.\mathbf{M}^{+}-57,100 \%\right)$. HRMS Calcd for $\mathrm{C}_{20} \mathrm{H}_{30} \mathrm{OSSi}$ : 346.1787. Found: 346.1788. 1Phenylthio-1-\{1-(t-butyldimethylsilyloxy)cyclohexyl\}ethene. Obtained in $99 \%$ yield. Colorless oil. ${ }^{1} \mathrm{H}-\mathrm{NMR}\left(400 \mathrm{MHz}, \mathrm{CDCl}_{3}\right) \delta 0.15(6 \mathrm{H}, \mathrm{s}), 0.94(9 \mathrm{H}, \mathrm{s}), 1.25-1.33(1 \mathrm{H}, \mathrm{m}), 1.47-1.55(3 \mathrm{H}, \mathrm{m})$, 1.63-1.73 (2H, m), $1.79(2 \mathrm{H}, \mathrm{td}, \mathrm{J}=13.2,3.6 \mathrm{~Hz}), 1.90-1.97(2 \mathrm{H}, \mathrm{m}), 4.55(1 \mathrm{H}, \mathrm{s}), 5.29(1 \mathrm{H}, \mathrm{s}), 7.31$ $(1 \mathrm{H}, \mathrm{t}, \mathrm{J}=7.6 \mathrm{~Hz}), 7.34(2 \mathrm{H}, \mathrm{t}, \mathrm{J}=7.2 \mathrm{~Hz}), 7.47(2 \mathrm{H}, \mathrm{dd}, \mathrm{J}=7.6,1.6 \mathrm{~Hz}) .{ }^{13} \mathrm{C}-\mathrm{NMR}(100 \mathrm{MHz}$, $\left.\mathrm{CDCl}_{3}\right) \delta-2.1,18.9,22.8,26.0,26.3,38.4,76.5,108.9,128.0,129.1,133.4,134.8,155.2$. IR (neat) 2924, 1602, 1470, 1255, 1145, 1022, 836, $773 \mathrm{~cm}^{-1}$. MS (EI) m/z $\mathbf{M}^{+}$, not detected. $333\left(\mathbf{M}^{+}-15,2 \%\right)$, $291\left(\mathbf{M}^{+}-57,100 \%\right)$. HRMS Calcd for $\mathrm{C}_{20} \mathrm{H}_{32} \mathrm{OSSi}$ : 348.1943, not detected. Calcd for $\mathrm{C}_{20} \mathrm{H}_{32} \mathrm{OSSi}-$ $\mathrm{C}_{4} \mathrm{H}_{9}: 291.1239$. Found: 291.1249 .

Reaction of 1-Octanethiol and 2-\{2-(t-Butoxycarbonylamino)ethylthio\}-1triisopropylsilylethyne 6. In a two-necked flask equipped with a reflux condenser were placed tetrakis(triphenylphosphine)hydriderhodium (4 mol\%, $6.9 \mathrm{mg})$, dppf (6 mol\%, $5.0 \mathrm{mg}), 6$ (0.15 mmol, $54 \mathrm{mg})$, and 1-octanethiol $(0.15 \mathrm{mmol}, 21.9 \mathrm{mg})$ in acetone $(2.0 \mathrm{~mL})$ under an argon atmosphere. The solution was heated at reflux for $1 \mathrm{~h}$. Then, the solvent was removed under reduced pressure, and the residue was purified by flash column chromatography on silica gel giving triisopropylsilylacetylene 7 (4.9 mg, 18\%), 1-octylthio-2-triisopropylsilylethyne 8 (13.4 mg, 27\%), 4 (11.5 mg, 43\%), dioctyl disulfide (4.6 mg, $0.016 \mathrm{mmol})$, octyl 2-( $t$-butoxycarbonylamino)ethyl disulfide ( $8.0 \mathrm{mg}, 0.025 \mathrm{mmol})$, and bis $\{2$-( $t$-butoxycarbonylamino)ethyl $\}$ disulfide $(0.7 \mathrm{mg}, 0.002 \mathrm{mmol})$ with recovered 1octanethiol (7.3 mg, 30\%) and 6 (22.1 mg, 41\%).

Alkylthio Exchange Reaction of 1-Alkylthio-1-alkyne and Disulfide. In a two-necked flask equipped with a reflux condenser were placed tetrakis(triphenylphosphine)hydriderhodium ( 2 mol\%, $5.8 \mathrm{mg}$ ), dppf (4 mol\%, $5.5 \mathrm{mg})$, 1-butylthio-2-triethylsilylethyne 9 (0.25 mmol, $57 \mathrm{mg}$ ), and bis(4methoxybutylthio) disulfide $(0.75 \mathrm{mmol}, 178.5 \mathrm{mg})$ in acetone $(0.5 \mathrm{~mL})$ under an argon atmosphere. The solution was heated at reflux for $0.5 \mathrm{~h}$. Then, the solvent was removed under reduced pressure, and the residue was purified by flash column chromatography on silica gel giving 1-(4methoxybutylthio)-2-triethylsilylethyne (51.6 mg, 80\%), butyl 4-methoxybutyl disulfide (35.4 mg, $68 \%)$ as well as recovered $9(7.4 \mathrm{mg}, 13 \%)$ and disulfide $(126.7 \mathrm{mg}, 71 \%)$.

\section{References}

(10) Ahmad, N.; Levison, J. J.; Robinson, S. D.; Uttley, M. F. Inorg. Synth. 1974, 15, 58.

(11) Moree, W. J.; van Gent, L. C.; van der Marel, G. A.; Liskamp, R. M. J. Tetrahedron, 1993, 49, 1133. 